Pierre-Alain Duc, Jonathan Braine and Elias Brinks, eds.

\title{
HST Observations of the Toomre Sequence of Merging Galaxies
}

Jörn Rossa ${ }^{1}$, Roeland P. van der Marel ${ }^{1}$, Torsten Böker ${ }^{1}$, Seppo Laine ${ }^{2}$, J. Christopher Mihos ${ }^{3}$, John E. Hibbard ${ }^{4}$, Ann I. Zabludoff ${ }^{5}$

${ }^{1}$ Space Telescope Science Institute, Baltimore, MD 21218, USA

${ }^{2}$ SIRTF Science Center, CALTECH, Pasadena, CA 91125, USA

${ }^{3}$ Case Western Reserve University, Cleveland, $O H$ 44106, USA

${ }^{4}$ NRAO, Charlottesville, VA 22903-2475, USA

${ }^{5}$ Steward Observatory, University of Arizona, Tucson, AZ 85721, USA

\begin{abstract}
.
We discuss our ongoing multi-instrument HST investigation of the nuclear regions of the 11 interacting and merging galaxies in the Toomre Sequence. We are studying the nuclear kinematics using STIS (G750M) spectra, the nuclear stellar populations using STIS (G430L) spectra, and the nuclear morphology using NICMOS and WFPC2 images. The results will provide new insight into the physical processes that operate during galaxy interactions.
\end{abstract}

\section{Introduction}

It is a generally accepted fact that galaxy interactions can trigger a burst of intense star formation. Various evolutionary aspects can be studied best in merging galaxies. Many galaxies of the Toomre Sequence (Toomre 1977) feature prominent tidal tails and complex structures on large scales. However, physical processes that operate during galaxy interactions often leave their most important signatures in the very central region. It is in these violent regimes where the recycling processes of the ISM can be investigated in great detail. The Toomre galaxies show abundantly distributed ionized gas which is the result of strong star formation activity. In turn, the star formation is due to gas flows to the center triggered by the merging process [cf. Schweizer (1998) for an overview]. HST allows us to investigate and quantify these processes at high spatial resolution.

\section{Observations and results}

We have used the WFPC2 for optical broad-band and emission-line imaging, and we see a slight trend of increasing luminosity density towards the latest merger stages within $100 \mathrm{pc}$ and $1 \mathrm{kpc}$ (Laine et al. 2003). However, NIR images are required to peer through the dust that is present in many of the galaxies. NICMOS K-band images reveal both nuclei of NGC 520, whereas optical images only show one. In NGC 7764A we detect a double nucleus in the NIR. The STIS kinematics reveal for late-type mergers a more or less regular rotation curve (see 

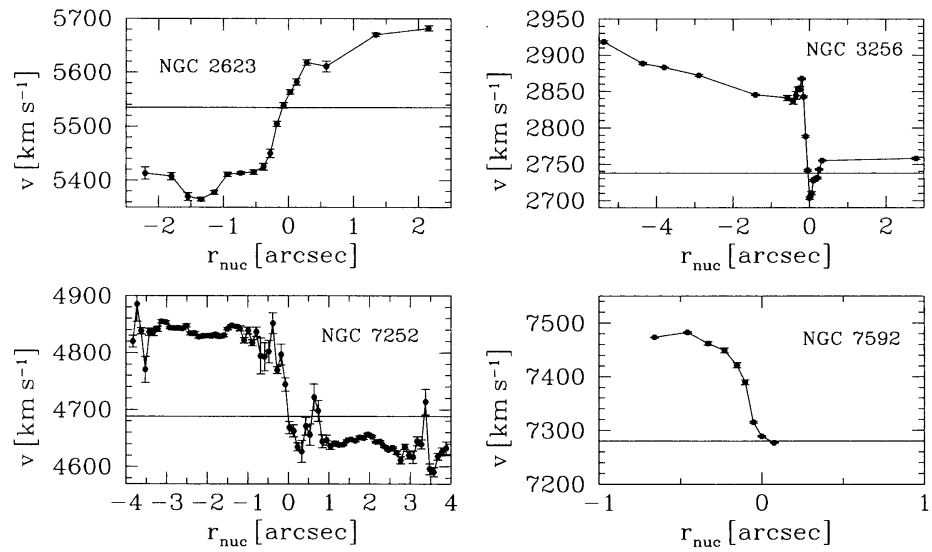

Figure 1. Nuclear $\mathrm{H} \alpha+[\mathrm{N}$ II $]$ rotation curves of four Toomre Sequence galaxies, derived from our STIS G750M observations. The solid horizontal line marks the $\mathrm{H}$ I systemic velocity. Note, that only NGC 7592 is an early-type merger.

Fig. 1), and generally a line broadening towards the central regions is found (velocity dispersions of $\sigma \approx 250-300 \mathrm{~km} \mathrm{~s}^{-1}$ are observed). Low-resolution STIS spectra are being fitted with stellar population synthesis models to study stellar population characteristics and ages. The results will be quantified both as a function of nuclear radial distance and merger stage within the Toomre Sequence. The combined data set will allow us to assess whether the Toomre Sequence forms an evolutionary sequence, and to study in detail the physical processes involved.

Acknowledgments. This contribution was partly supported by a travel grant from the AAS. The project is based on observations made with the NASA ESA Hubble Space Telescope, obtained [from the Data Archive] at the Space Telescope Science Institute, which is operated by the Association of Universities for Research in Astronomy, Inc., under NASA contract NAS 5-26555, and was supported by NASA/STScI grants for programs GO-8669 and GO-9402.

\section{References}

Laine, S., van der Marel, R. P., Rossa, J., Böker, T., Mihos, J. C., Hibbard, J. E., \& Zabludoff A. I. 2003, AJ, 126, 2717

Schweizer, F. 1998, in: Galaxies: Interactions and Induced Star Formation, Saas Fee Advanced Course 26, ed. D. Friedli, L. Martinet \& D. Pfenniger, (Berlin: Springer Verlag), 105

Toomre, A. 1977, in: The Evolution of Galaxies and Stellar Populations, ed. B.M. Tinsley \& R.B. Larson (New Haven: Yale Univ.), 401 